\title{
Clinical characteristics of glioblastoma with metastatic spinal dissemination
}

\author{
Jianxin Chen", Qi Shi", Shan Li, Yuze Zhao, Hongyan Huang^^ \\ Department of Oncology, Beijing Shijitan Hospital of Capital Medical University, Beijing, China \\ Contributions: (I) Conception and design: J Chen, H Huang; (II) Administrative support: H Huang; (III) Provision of study materials or patients: \\ J Chen, Q Shi; (IV) Collection and assembly of data: J Chen, Q Shi, S Li, Y Zhao; (V) Data analysis and interpretation: Q Shi, H Huang; (VI) \\ Manuscript writing: All authors; (VII) Final approval of manuscript: All authors. \\ \#Theses authors contributed equally to this work. \\ Correspondence to: Hongyan Huang. Department of Oncology, Beijing Shijitan Hospital of Capital Medical University, Beijing, China. \\ Email: huangh1975@mail.ccmu.edu.cn.
}

Background Metastatic spinal dissemination (MSD) is a rare phenomenon in glioblastoma (GBM). The study aimed to analyze the clinical characteristics of GBM patients with MSD.

Methods: Fifteen GBM patients with MSD, who were treated and followed up with at the Department of Oncology, Beijing Shijitan Hospital, Capital Medical University from September 2012 to February 2021, were selected for this study. Clinical data, such as demographic characteristics, clinical manifestation, imaging, cerebrospinal fluid (CSF), treatment and prognosis data, were retrospectively analyzed. The time to MSD and overall survival (OS) were estimated using Kaplan-Meier plotting. A univariate analysis was performed using a logarithmic-rank test, and a multivariate analysis was performed using Cox proportional hazards models.

Results: Of the 15 GBM patients with MSD (9 males and 6 females), the primary lesions were located supratentorial region in 12 cases, and subtentorial region in 3 cases. After surgery, the ventricles were open and closed in 7 and 8 cases, respectively. There were 10 cases, 2 cases, 2 cases, and 1 case of MSD in the full spinal cord (FSC), FSC with spinal cord intramedullary infiltration, cervical spinal cord intramedullary infiltration, and cervical/thoracic MSD, respectively. Whole spinal cord magnetic resonance imaging (MRI) showed dotted-line, nodules, and mixed patterns in 3, 2, and 10 cases, respectively. The average CSF protein level during MSD was 2.49 (range, 0.42-6.68) g/L, and the average CSF d-dimer level was 23,718 (range, 4,056-69,000) ng/L. By the end of the follow-up period, all the patients had died. The median OS of all patients, the median time from surgery to diagnosis of MSD, and the median time after MSD to death was 15.0 (range, 8-52), 10.0 (range, 1-49), and 4.0 (range, 1-14) months, respectively.

Conclusions: MSD is a rare, metastasized type of GBM. The OS after MSD in GBM patients is very short. Whole spinal cord-enhanced MRI may be the best way to determine the range of MSD.

Keywords: Glioblastoma (GBM); metastatic spinal dissemination (MSD); clinical characteristics; prognosis

Submitted Oct 29, 2021. Accepted for publication Dec 30, 2021.

doi: 10.21037/apm-21-3387

View this article at: https://dx.doi.org/10.21037/apm-21-3387

^ ORCID: 0000-0001-8340-6660. 


\section{Introduction}

Glioblastoma (GBM) is the most common and least prognostic malignant glioma of primary origin in the central nervous system (CNS). It has a median survival time of 14.6 months after standard treatment with the Stupp regimen, and the vast majority of patients die due to progression of tumor recurrence $(1,2)$. A proportion of $80 \%$ of patients have recurrence in situ, $21.0 \%$ have distant metastases, and a few have metastatic spinal dissemination (MSD) (3). MSD occurs at every stage of the disease, mainly through the cerebrospinal fluid (CSF) (4). As cases of MSD in GBM have rarely been reported (and most reports are case reports), the clinical features of MSD are poorly understood $(5,6)$. In our institution, the incidence of MSD in GBM patients was 3-5\%. The study is to further clarify the general clinical characteristics of glioblastoma with metastatic spinal dissemination in China and sought to summarize the clinical features of patients with MSD in GBM to improve the clinical management of MSD. We present the following article in accordance with the STROBE reporting checklist (available at https://apm. amegroups.com/article/view/10.21037/apm-21-3387/rc).

\section{Methods}

\section{Patients and study design}

Fifteen GBM patients with MSD were treated at Beijing Shijitan Hospital from September 2012 to February 2021 with complete follow-up. All patients were pathologically diagnosed with GBM in the primary lesion tissue, and the pathological diagnosis was made using the 2007 World Health Organization (WHO) classification and diagnostic criteria for CNS malignancies (7). Clinical data on clinical symptoms, previous treatment, primary tumor site, postdiffusion treatment, and survival were retrospectively collected.

Confirmation of MSD includes the detection of tumor cells on CSF cytology or whole spinal cord-enhanced magnetic resonance imaging (MRI) showing abnormally enhanced lesions at the periphery of the corresponding spinal cord segment or within the spinal cord. MSD lesion imaging shows three patterns: (I) dotted-line; (II) nodular, and (III) mixed patterns (8). CSF cytology and protein quantification were routinely performed in the patients with confirmed MSD, and D-dimer (D-Dimer) levels were measured.

The follow-up appointments were primarily conducted by outpatient and inpatient reviews or telephone visits.

\section{Statistical analysis}

Overall survival (OS) was defined as the time from surgery to the death of the patient. Survival after MSD was defined as the time from diagnosis of MSD to death of the patient. Time to MSD was defined as the time from initial surgery to confirmed MSD. Survival curves were plotted using the Kaplan-Meier method. All analyses were performed with SPSS (version 23, IBM Corporation, Armonk, NY, USA). A $\mathrm{P}$ value $<0.05$ was considered statistically significant.

\section{Ethical statement}

All procedures performed in this study involving human participants were in accordance with the Declaration of Helsinki (as revised in 2013). The study was approved by institutional ethics board of Beijing Shijitan Hospital [No. sjtky11-lx-2021(64)]. Individual consent for this retrospective analysis was waived.

\section{Results}

\section{Patients' characteristics}

From September 2012 to February 2021, 15 patients with GBM MSD were enrolled in this retrospective study. The characteristics of the 15 patients are summarized in Table 1 . Of the 15 patients, 9 were male and 6 were female. The patients had a median age of 37 (range, 21-78) years. The primary lesion was located supratentorially in 12 cases (5 frontal, 3 temporal, 2 parieto-occipital, and 2 temporooccipital), and there were 2 cases of invasion of the corpus callosum. All 15 patients underwent surgery. Specifically, 12 patients underwent total or near-total resection, and 3 patients underwent partial resection. The ventricles were open in 7 cases and closed in 8 cases after surgery. All patients received concurrent radiotherapy after surgery, and 14 patients received temozolomide (TMZ) after radiotherapy (5/28 regimen), with a median of 6 (range, 1-24) cycles of adjuvant chemotherapy.

\section{Clinical manifestations of MSD}

Thirteen GBM patients with MSD presented with single or multiple symptoms, including lower extremity dyskinesia, lower extremity pain, neck pain, lower back pain, lower 
Table 1 Patients' demographic characteristics

\begin{tabular}{|c|c|c|c|c|c|c|}
\hline Pt. No. & Sex & Age (years) & Primary tumor location & Surgery & $\begin{array}{l}\text { Postoperative } \\
\text { ventricular open }\end{array}$ & Symptoms of MSD \\
\hline 1 & $\mathrm{~F}$ & 21 & B (brainstem) & PR & $\mathrm{N}$ & Back pain \& dyskinesia \\
\hline 3 & $\mathrm{~F}$ & 28 & B (L temporal-occipital) & GTR & Y & Headaches and foot pain \& dyskinesia \\
\hline 6 & M & 37 & B (R temporal) & GTR & $\mathrm{N}$ & Numbness of the right lower limb with dyskinesia \\
\hline 7 & M & 27 & B (R parietal-occipital) & GTR & $\mathrm{N}$ & Headaches \& dyskinesia \\
\hline 8 & M & 33 & B (L occipital) & GTR & $\mathrm{N}$ & Neck pain \& dysuria \\
\hline 12 & M & 54 & $B(L$ frontal) & GTR & $\mathrm{Y}$ & Neck and shoulder pain \\
\hline 13 & $\mathrm{~F}$ & 62 & $B$ (L frontal) & PR & $\mathrm{N}$ & Drowsiness, epilepsy, dyskinesia of lower limb \\
\hline 14 & $\mathrm{~F}$ & 29 & $S(C 1-7)$ & GTR & Y & Urinary incontinence \& epilepsy \\
\hline 15 & $\mathrm{~F}$ & 78 & $\mathrm{~B}(\mathrm{~L}$ temporal) & GTR & $\mathrm{N}$ & Dyskinesia \& consciousness \\
\hline
\end{tabular}

Pt, patient; No., number; F, female; M, male; B, brain; S, spine; L, left; R, right; C cervical; PR, partial resection; GTR, gross-total resection; $\mathrm{N}, \mathrm{No}$; Y, yes; MSD, metastatic spinal dissemination; MRI, magnetic resonance imaging.

extremity numbness, urinary retention/incontinence, impaired consciousness, gait abnormalities, headache, and epilepsy. Lower limb dyskinesia was the most common symptom (11/15), followed by neck and lower back pain in 4 patients, and urinary incontinence/retention in 3 patients. Two patients were asymptomatic at the time of detection of MSD.

Enhanced MRI of the whole spinal cord revealed 12 MSD cases in total, including 2 cases with intramedullary invasion of the spinal cord. There was 1 case of cervicothoracic MSD and 2 cases of intramedullary invasion of the cervical medulla. Enhanced MRI of the spinal cord showed disseminated lesions of the dotted-line, nodular, and mixed pattern types in 3, 2, and 10 cases, respectively.

In 14 patients, no tumor cells were detected by CSF cytology, and in 1 case were not tested. Ten patients had CSF protein levels above the upper limit of normal, 1 case had normal CSF protein levels, and in 4 cases, CSF protein levels were not detected. Patients had a mean protein level of 2.49 (range, 0.42-6.68) $\mathrm{g} / \mathrm{L}$. Of the 15 patients, 8 had mean CSF D-Dimer levels of 23,718.0 (range, 4,056-69,000) ng/L.

After the MSD diagnosis, a part of patients received surgery. 1 patient received total craniospinal radiotherapy, and 2 patients received local radiotherapy to the affected spinal cord segments. Eleven patients received single/2drug combination chemotherapy or targeted therapy with TMZ, etoposide, carboplatin, cisplatin, irinotecan, sotan, lomustine, teniposide, cyclophosphamide, verofenib, darafenib, bevacizumab, and enduro, 6 patients received intrathecal methotrexate, and 4 patients received symptomatic supportive therapy only. The efficiency of the treatment was little, and we provided representative images of MRI before and after treatment about MSD in GBM (see Figure 1).

\section{Follow-up}

By February 2021, all 15 patients had died. The median OS was 15.0 (range, 8-52) months (see Figure 2). The median time from initial surgery to MSD was 10.0 (range, 1-49) months (see Figure 3). The median survival time after MSD was 4.0 (range, 1-14) months (see Figure 4). The median OS time, median time from initial surgery to MSD, and median survival time after MSD in the 6 cases with intracranial first 

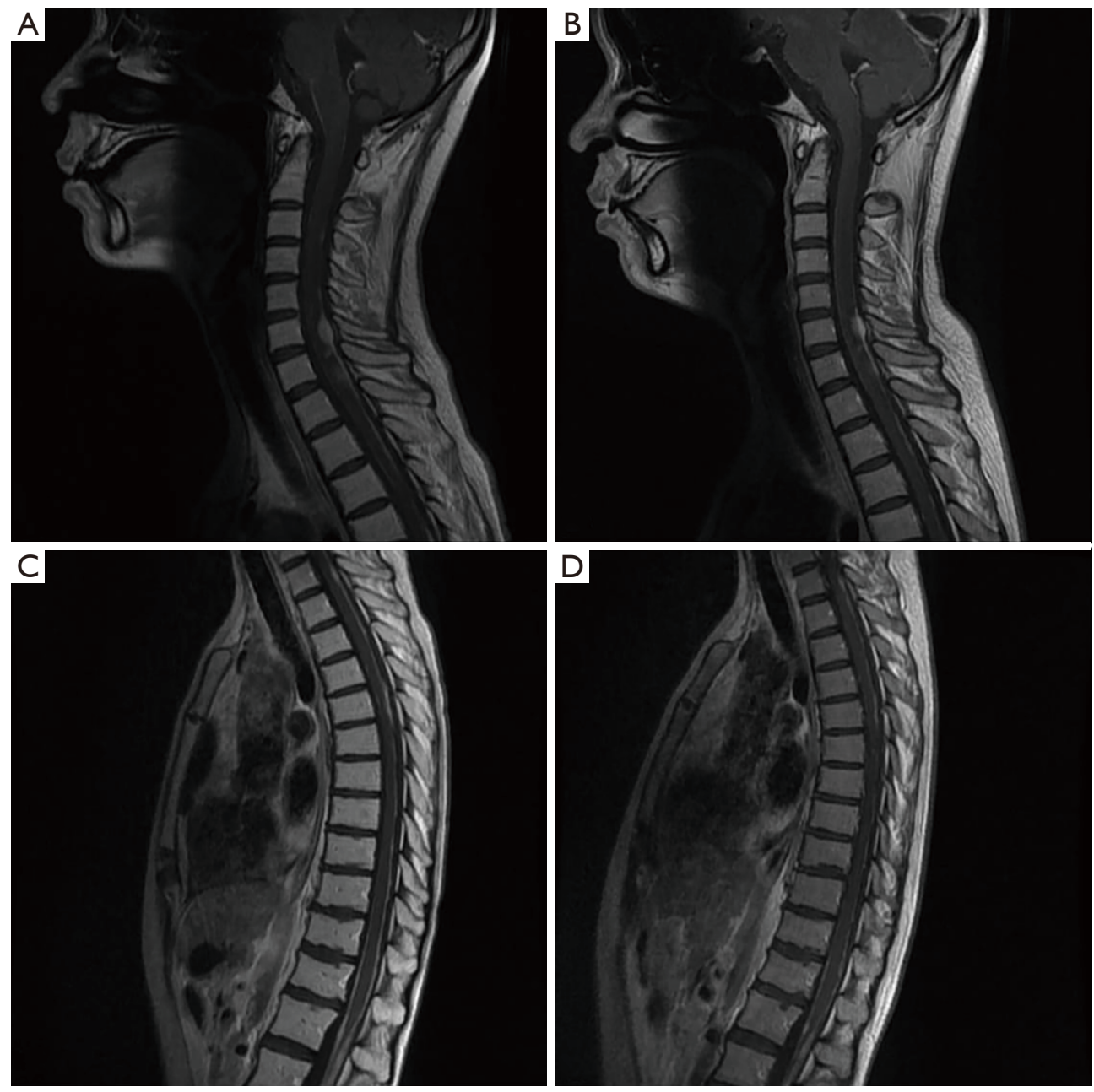

Figure 1 One patient (female, 28 year-old) in this study with MSD about cervical and thoracic spinal spread, respectively. (A,C) Metastatic spinal dissemination before treatment. (B,D) Metastatic spinal dissemination after treatment. MSD, metastatic spinal dissemination.

recurrence was 18.0 (range, 9-52), 15.5 (range, 10-49), and 2.0 (range, 1-6) months, respectively. Two patients with spinal cord first recurrence and 7 patients with concurrent spinal cord intracranial recurrence had a median OS time, median time from initial surgery to MSD, and median survival time after MSD of 14.0 (range, 8-32), 7.0 (range, 1-22), and 5.0 (range, 1-12) months, respectively.

\section{Discussion}

GBM accounts for $54 \%$ of intracranial primary brain malignant glioma (9). Recently, due to the increasing incidence of glioma and advances in examination techniques, the number of reports of GBM patients with MSD has gradually increased. In this study, the clinical characteristics of 15 GBM patients with MSD were reviewed and analyzed. The clinical manifestations of these patients mainly included the compression of the affected segments of the spinal cord, resulting in corresponding dysfunction, which often manifested as lower limb movement disorders, pain, and abnormal urination. As MSD usually occurs simultaneously or sequentially with the progression of intracranial GBM, patients are often asymptomatic, or the symptoms appear late. Indeed, as most symptoms occur in the late stage of the disease, the progression of intracranial GBM leading to motor disorders and sensory abnormalities may mask the symptoms of MSD. When intracranial GBM recurrence presents with clinical symptoms that cannot be explained by intracranial GBM lesions and the progress is rapid, the possibility of MSD should be considered. In this 


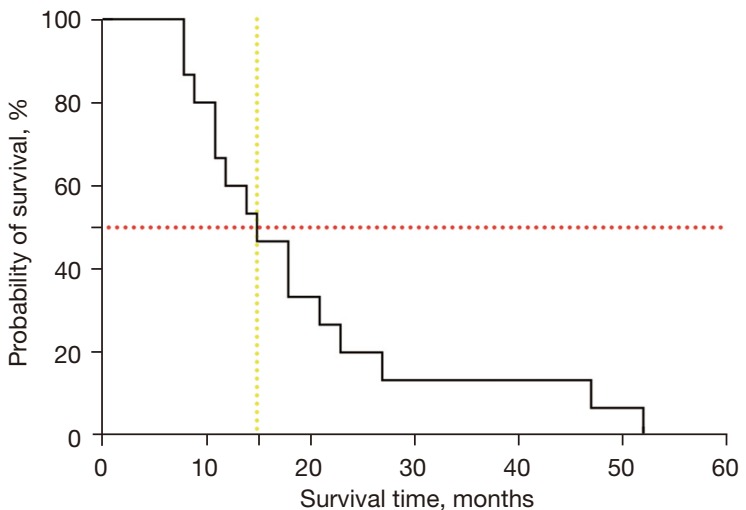

Figure 2 Median overall survival time (15 months). A Kaplan-Meier analysis was conducted to calculate the median time.

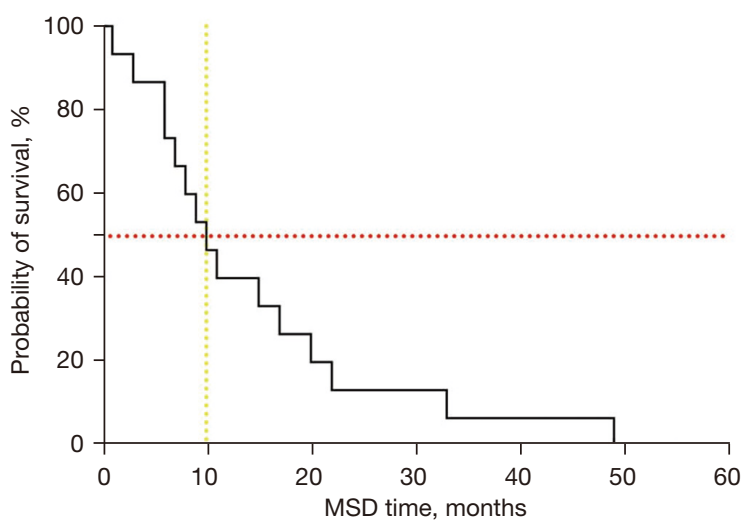

Figure 3 Median time to metastatic spinal dissemination (10 months). A Kaplan-Meier analysis was conducted to calculate the median time. MSD, metastatic spinal dissemination.

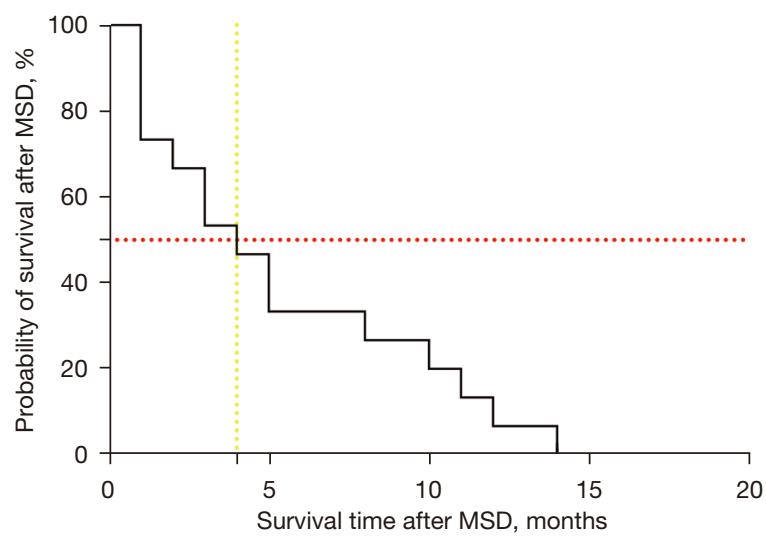

Figure 4 Median survival time after metastatic spinal dissemination (4 months). A Kaplan-Meier analysis was conducted to calculate the median time. MSD, metastatic spinal dissemination. study, two patients presented with MSD without obvious clinical symptoms, suggesting that the occurrence of MSD is insidious to some extent. Whole spinal cord-enhanced MRI can help to detect MSD. Otherwise, the time point of the whole spinal cord test from large-scale clinical studies on the target population is still pending (10).

The diagnostic criteria for MSD include CSF cytology and whole spinal cord-enhanced MRI. The finding of tumor cells in the CSF is the highest criterion for a diagnosis of MSD in GBM, but the chances of positive cytology are low. In the 14 cases observed in this study, the CSF cytology was negative. Thus, whole spinal cord-enhanced MRI is currently the most effective means of diagnosing MSD in clinical practice. We also measured protein levels and D-Dimer in the CSF, and the results showed that $90.9 \%$ (10/11) of patients with MSD had varying degrees of elevated CSF protein levels, but there was a wide variation in the magnitude of elevation. In this study, eight patients had CSF D-Dimer levels that exceeded the upper limit of normal plasma levels by more than 10-100 times. Thus, increased CSF D-Dimer levels may be associated with the occurrence of MSD. However, the question of whether CSF D-Dimer levels can be used as a biomarker for GBM MSD needs to be further investigated with a larger sample size.

MSD is a rare form of GBM metastasis (4,5,11-13). There is no standard recommendation for the treatment of MSD; the available treatment options include radiotherapy, chemotherapy, intrathecal injection of chemotherapeutic agents, and targeted drug therapy. Despite these treatments, the mean survival of patients with MSD in the present study was only 4 months; $66.7 \%$ (10 cases) survived $<5$ months, and $93.3 \%$ (14 cases) survived $<1$ year. In the present study, 1 patient with primary cervical GBM, who received systemic therapy with irinotecan, Endo, and carboplatin after the diagnosis of MSD, survived 14 months after MSD, which suggests that chemotherapy combined with targeted therapy may contribute to prolonging patient survival. However, new clinical studies need to be designed and conducted to determine how to improve patient prognosis through treatment.

The mechanisms and risk factors of MSD in GBM patients remain unclear. The main causes of MSD are the metastasis of GBM cells with CSF, inadequate surgical protection, open ventriculus cerebri, or the proximity of the tumor to the ventriculus cerebri (14). Scoccianti reported that risk factors for spinal dissemination include being male, the surgical opening of the ventricles and multiple 
foci (15). Tsung concluded that repeated surgical resection, male, ventricular invasion, ventricular epithelial rupture, and decreased immunity can increase the incidence of spinal dissemination. Tsung suggested that repeated surgical resection, ventricular invasion, ventricular epithelial rupture, and decreased immune function could increase the incidence of MSD (16). Wright reported that MSD occurred in all cases of ventricular opening after GBM and in no cases without ventricular opening (17). Thus, the ventricular opening, subventricular infiltration, and the proximity of the tumor to the ventricles may be predictors of MSD (15). Fifteen MSD cases were reported in this study, of which 7 had a ventricular opening and 9 had subventricular infiltration. Thus, subventricular infiltration or a ventricular opening may increase the risk of MSD. Further research needs to be conducted on the risk factors for the occurrence of MSD during the treatment of GBM to avoid the poor prognosis caused by MSD as much as possible.

Several factors limited this retrospective research, including its small sample size. A small number of the sample reached the survival event, and in future, we will accumulate more cases to further explanation.

In summary, MSD is a relatively rare form of metastasis from GBM, and the molecular mechanisms by which it occurs remain unclear. Its diagnosis relies mainly on positive findings from whole spinal cord-enhanced MRI scans, and its treatment lacks effective therapeutic tools, resulting in a poor overall prognosis. The development of safe and effective treatment regimens and the exploration of markers associated with the progression of MSD are key directions for future research.

\section{Acknowledgments}

Funding: This work was funded by the National Natural Science Foundation of China (No. 81572799).

\section{Footnote}

Reporting Checklist: The authors have completed the STROBE reporting checklist. Available at https://apm. amegroups.com/article/view/10.21037/apm-21-3387/rc

Data Sharing Statement: Available at https://apm.amegroups. com/article/view/10.21037/apm-21-3387/dss

Conflicts of Interest: All authors have completed the
ICMJE uniform disclosure form (available at https://apm. amegroups.com/article/view/10.21037/apm-21-3387/coif). The authors have no conflicts of interest to declare.

Ethical Statement: The authors are accountable for all aspects of the work in ensuring that questions related to the accuracy or integrity of any part of the work are appropriately investigated and resolved. All procedures performed in this study involving human participants were in accordance with the Declaration of Helsinki (as revised in 2013). The Institutional Ethics Committee of the Beijing Shijitan Hospital approved this study [No. sjtky11$1 \mathrm{x}$-2021(64)]. Written informed consent was waived for the study due to its retrospective nature.

Open Access Statement: This is an Open Access article distributed in accordance with the Creative Commons Attribution-NonCommercial-NoDerivs 4.0 International License (CC BY-NC-ND 4.0), which permits the noncommercial replication and distribution of the article with the strict proviso that no changes or edits are made and the original work is properly cited (including links to both the formal publication through the relevant DOI and the license). See: https://creativecommons.org/licenses/by-nc-nd/4.0/.

\section{References}

1. Stupp R, Mason WP, van den Bent MJ, et al. Radiotherapy plus concomitant and adjuvant temozolomide for glioblastoma. N Engl J Med 2005;352:987-96.

2. Brandes AA, Tosoni A, Franceschi E, et al. Recurrence pattern after temozolomide concomitant with and adjuvant to radiotherapy in newly diagnosed patients with glioblastoma: correlation With MGMT promoter methylation status. J Clin Oncol 2009;27:1275-9.

3. Milano MT, Okunieff P, Donatello RS, et al. Patterns and timing of recurrence after temozolomide-based chemoradiation for glioblastoma. Int J Radiat Oncol Biol Phys 2010;78:1147-55.

4. Arita N, Taneda M, Hayakawa T. Leptomeningeal dissemination of malignant gliomas. Incidence, diagnosis and outcome. Acta Neurochir (Wien) 1994;126:84-92.

5. Bae JS, Yang SH, Yoon WS, et al. The clinical features of spinal leptomeningeal dissemination from malignant gliomas. J Korean Neurosurg Soc 2011;49:334-8.

6. Shahideh M, Fallah A, Munoz DG, et al. Systematic review of primary intracranial glioblastoma multiforme with symptomatic spinal metastases, with two illustrative 
patients. J Clin Neurosci 2012;19:1080-6.

7. Louis DN, Ohgaki H, Wiestler OD, et al. The 2007 WHO classification of tumours of the central nervous system. Acta Neuropathol 2007;114:97-109.

8. Lam CH, Cosgrove GR, Drislane FW, et al. Spinal leptomeningeal metastasis from cerebral glioblastoma. Appearance on magnetic resonance imaging. Surg Neurol 1991;35:377-80.

9. Ostrom QT, Gittleman H, Farah P, et al. CBTRUS statistical report: Primary brain and central nervous system tumors diagnosed in the United States in 2006-2010. Neuro Oncol 2013;15 Suppl 2:ii1-56.

10. Birbilis TA, Matis GK, Eleftheriadis SG, et al. Spinal metastasis of glioblastoma multiforme: an uncommon suspect? Spine (Phila Pa 1976) 2010;35:E264-9.

11. Lucas J, Zada G. Radiology: criteria for determining response to treatment and recurrence of high-grade gliomas. Neurosurg Clin N Am 2012;23:269-76, viii.

12. Choucair AK, Levin VA, Gutin PH, et al. Development of multiple lesions during radiation therapy and chemotherapy in patients with gliomas. J Neurosurg

Cite this article as: Chen J, Shi Q, Li S, Zhao Y, Huang H. Clinical characteristics of glioblastoma with metastatic spinal dissemination. Ann Palliat Med 2022;11(2):506-512. doi: 10.21037/ apm-21-3387
1986;65:654-8.

13. Vertosick FT Jr, Selker RG. Brain stem and spinal metastases of supratentorial glioblastoma multiforme: a clinical series. Neurosurgery 1990;27:516-21; discussion 521-2.

14. Vollmer K, Pantazis G, Añon J, et al. Spinal Metastases of Supratentorial Glioblastoma with Primitive Neuronal Component. World Neurosurg X 2019;2:100019.

15. Scoccianti S, Detti B, Meattini I, et al. Symptomatic leptomeningeal and intramedullary metastases from intracranial glioblastoma multiforme: a case report. Tumori 2008;94:877-81.

16. Tsung AJ, Prabhu SS, Lei X, et al. Cerebellar glioblastoma: a retrospective review of 21 patients at a single institution. J Neurooncol 2011;105:555-62.

17. Wright CH, Wright J, Onyewadume L, et al. Diagnosis, treatment, and survival in spinal dissemination of primary intracranial glioblastoma: systematic literature review. J Neurosurg Spine 2019. [Epub ahead of print].

(English Language Editor: L. Huleatt) 